\title{
Emerging Technologies for Diabetes Care
}

\author{
Timothy S. Bailey, MD, FACE, FACP, CPI, John Walsh, PA, CDTC, , and Jenine Y. Stone, FNP-C ${ }^{1}$
}

\begin{abstract}
New therapies, monitoring, and revolutionary enabling technologies applied to healthcare represent an historic opportunity to improve the lives of people with diabetes. These advances enable more meaningful monitoring of blood glucose values with the facilitation of more optimal insulin dosing and delivery. Newer insulins and delivery systems are in development that seek to mitigate both hyperglycemia and hypoglycemia and increase time in range. Information systems now exist that may be leveraged to merge data from previously discrete systems into new models of connected care. This review highlights important developments that serve to increase effectiveness while reducing the burden of diabetes care in the near future.
\end{abstract}

Keywords: Diabetes technology, Glucose sensors, Connected care, Computer decision support, Insulin pumps, Insulin therapy.

\section{Introduction}

D IABETES IS A COMPLEX, chronic condition estimated in 2014 by the World Health Organization to affect 422 million people globally. ${ }^{1}$ Despite significant therapeutic advancements, a person with diabetes routinely experiences physiological, cognitive, pragmatic, and psychological burdens. To achieve and maintain optimal glycemic control, those who require insulin generally must engage in timeconsuming behaviors such as frequent glucose monitoring (GM) and quantifying ("counting") carbohydrate intake, while also taking into consideration variables such as noncarbohydrate food content, exercise, illness, menstruation, stress, and other life events to adjust their medication doses. These ongoing efforts are undertaken while also attempting to avoid hypo- and hyperglycemia and lead normal lives. A person must not only have access to the right therapy (ideally individualized), but also be engaged in and adherent to a treatment plan that requires lifestyle changes to manage this complex disease. Many fail to achieve their glycemic goals due to multiple factors, including delays in intensification of treatment regimens (on the part of the patient as well as the clinician) ${ }^{2}$ resistance to changes in lifestyle ${ }^{3,4}$ lack of patient education resources, inadequate treatment regimens, and poor adherence to treatment.

"Optimal glycemic control" is now understood to be more than simply achieving a target hemoglobin A1c (HbA1c) level. Other factors such as glycemic variability and "time-in-range" may have important clinical implications in cardiovascular and other outcomes. ${ }^{7-9}$ A given HbA1c can be associated with a wide range of mean glucose concentrations between individuals, and similarly misleading; a given mean glucose concentration has been shown to be associated with variable HbA1c values among different racial or ethnic groups. For these reasons, $\mathrm{HbAlc}$ is considered to be less reliable than direct calculation of mean blood glucose (using continuous glucose monitoring [CGM] data), especially for use in monitoring and adjustment of treatment plans. ${ }^{10}$ Metrics to characterize the quality of glycemic control are growing in number and importance. Given the labor-intensive and data-driven nature of diabetes management, having access to the right tools can be critical to improving glycemic control.

This review highlights emerging technologies in the fields of insulins, GM, medication delivery, data management, and decision analysis. It will additionally explore the importance of connected care and the changing roles required for the person with diabetes and the clinician who strives to provide their care.

\section{New and Smarter Insulins}

\section{Ultrarapid insulins}

Faster acting insulin analogues have been under development in recent years to improve postprandial (PP) glycemic control (i.e., prevent and/or reduce PP glycemic excursions). Their faster onset will also enable more rapid correction of hyperglycemia. Novo Nordisk received FDA approval in September 2017 for Fiasp (a new formulation of insulin

\footnotetext{
${ }^{1}$ AMCR Institute, Escondido, California.

${ }^{2}$ Advanced Metabolic Care + Research, Escondido, California.
} 
aspart). ${ }^{11,12}$ Fiasp is produced by adding the excipients niacinamide (vitamin B3) that helps to increase the speed of initial absorption and $\mathrm{L}$-arginine that serves as a stabilizing agent. ${ }^{13,14}$ In a pooled analysis of six trials evaluating the pharmacokinetic and pharmacodynamic characteristics of Fiasp in adults with type 1 diabetes, Fiasp consistently demonstrated earlier onset of appearance, and greater early insulin exposure and glucose-lowering effect than traditional insulin aspart across all trials. ${ }^{13}$ In phase III trials, Fiasp was shown to improve PP glucose control compared with insulin aspart after mixed meal tolerance testing in individuals with both type 1 and type 2 diabetes. ${ }^{15,16}$ The improved effect of Fiasp is also attributed to an earlier suppression of endogenous glucose. ${ }^{17}$

A clinical trial evaluating compatibility, efficacy, and safety of Fiasp compared with insulin aspart in continuous subcutaneous insulin infusion (CSII) demonstrated that Fiasp is comparably safe and compatible for use in CSII, with no new safety issues observed in either treatment arm. ${ }^{18}$ Other studies have demonstrated similarly improved pharmacokinetic (PK) and pharmacodynamic (PD) profiles ${ }^{19}$ and improved PP glucose control $^{20}$ for Fiasp versus insulin aspart in CSII usage, consistent with prior studies for multiple dose injection (MDI) users. ${ }^{15,16}$ Future trials will investigate the potential benefits of faster insulins in artificial pancreas (AP) systems.

An ultrarapid formulation of insulin lispro (Eli Lilly) is currently undergoing evaluation in phase III trials, with planned regulatory submission for $2019 .^{21}$ French biotechnology company Adocia is also continuing to develop an ultrarapid insulin formulation called BioChaperone Lispro, which should be ready to enter phase III trials soon. ${ }^{21}$

\section{"Smart" insulin}

In addition to algorithm-driven AP technologies, another approach to "glucose-responsive" insulin therapy has been through the development of "smart insulin." The concept is that insulin itself would sense and respond to glucose, activating release only when needed through methods such as glucoseresponsive polymer encapsulation or direct modification of the insulin molecule. ${ }^{22}$ JDRF (a charitable organization that funds type 1 diabetes research) was an early supporter of these efforts, investing in a company called SmartCells, Inc., founded in 2003, whose insulin product was engineered to become bioavailable only in the presence of elevated glucose. SmartCells was later acquired by Merck in 2010, and completed phase I trials in 2016, after which the development of the drug was terminated due to lack of efficacy observed in early trials. ${ }^{23}$

JDRF continues to support development of smart insulin products and has partnered with Sanofi to provide funding in support of basic research projects to determine the best approach going forward. ${ }^{23}$ Four researchers were awarded funding from the partnership in 2016, focusing on complementary approaches to the development of smart insulin. Two of the four groups are focused on biochemically engineered glucose-responsive insulin analogues, one of which is studying insulin derivatives that have been modified to provide both long-lasting activity (incorporating an aliphatic moiety to enable binding to serum albumin) and glucose-mediated activity (through incorporation of phenylboronic acid). These molecules are designed essentially to bind to albumin, and detach to activate in the presence of increased glucose concentrations. These have been verified in preclinical in vivo testing. ${ }^{24}$ Other groups are focused on glucose-sensing nanoparticles ${ }^{25}$ and glucose-responsive insulin delivery through polymer encapsulation ("artificial beta cells" that would be either implanted or delivered through a skin patch). ${ }^{26}$

\section{Inhaled insulins}

Subcutaneous insulin injections remain the primary route of insulin delivery despite being somewhat cumbersome and uncomfortable. Inhaled insulin had been an attractive "meal insulin" option due to the potential for elimination of inconvenient injections and for rapid insulin action. Despite its conceptual promise, efforts to realize common usage of inhaled insulin have not yet succeeded. Needle phobia has likely been overestimated, ${ }^{27}$ and development of molecules that can be adequately delivered and absorbed into the alveoli has been a challenge. ${ }^{28,29}$

Afrezza was FDA approved in 2014 and is currently the only inhaled insulin available on the market. ${ }^{30}$ Afrezza is recombinant human insulin that is adsorbed onto Technosphere particles, which are formed by the self-assembly of inert excipient fumaryl diketopiperazine. ${ }^{31,32}$ The size of the Technosphere particles enables delivery of insulin to the deep lung for better systemic absorption. ${ }^{31}$ Afrezza has a more rapid onset and shorter duration of action than subcutaneously injected rapidacting insulin analogues. In clinical trials, the median time to peak activity of Afrezza was shown to be $53 \mathrm{~min}$, and duration of action to be $\sim 160 \mathrm{~min}(2.5-3 \mathrm{~h}) .{ }^{33}$ Its use is associated with less frequent PP hypoglycemia and slightly less weight gain. ${ }^{34}$

Challenges with the clinical use of Afrezza include nonequivalency of doses compared with injections, cough, need for baseline and periodic assessment of lung function, possible variability in pulmonary absorption during respiratory infections, and cost. ${ }^{34}$ Afrezza has been associated with a small but persistent decrease in lung function as measured by Forced Expiratory Volume in one second (FEV1). It is thought that the observed decline in FEV1 is reversible after discontinuation of Afrezza. ${ }^{33}$ Dance Biopharm has developed an inhaled insulin system without excipients as a strategy to avoid changes in pulmonary function and cough; data are not available to confirm this. Owing to the short duration of action of Afrezza, additional doses may be required to maintain glycemia after certain meals (and, in particular, high fat meals). A trial investigating the role of CGM in directing additional doses of Afrezza postprandially is due to report soon. ${ }^{35}$

\section{Other noninjected therapies}

Although it offers a faster and more direct path to the portal vein and liver, challenges to the oral delivery of insulin primarily involve the ability of the molecule to survive the gastric environment while achieving appropriate absorption and distribution. These include physical barriers of the gastrointestinal tract, biochemical degradation by variations in recipients' gastric enzymes and $\mathrm{pH}$, and barriers resulting from the formulation of the drug. ${ }^{36}$ Although oral insulin therapies have yet to see progress, oral GLP-1 agonists show promise. Novo Nordisk is completing phase III trials of an oral formulation of semaglutide. ${ }^{37}$ This product is coformulated with sodium N (8 [2 hydroxybenzoyl] amino) caprylate, which facilitates absorption through the gastric epithelium by increasing local $\mathrm{pH} .{ }^{38}$ The phase II trials demonstrated improved A1c and weight reduction relative to placebo. ${ }^{39}$ 
Oramed is developing an oral formulation of exenatide (ORMD-0901) and is additionally seeking to combine it with insulin. $^{40}$

Intarcia Therapeutics, Inc. has developed an osmotic minipump (ITCA 650) that delivers exenatide for 6 months, and eventually 12 months. ${ }^{41}$ It is placed in the subdermal space by a minor office procedure. In a phase III trial of adults with uncontrolled type 2 diabetes on oral medications, ITCA 650 demonstrated superior weight and A1c reductions compared with placebo; nausea was the most common adverse event. ${ }^{42}$ This could address the low reported adherence and persistence with GLP-1 therapy. ${ }^{35}$

\section{Glucose Monitoring}

Despite the dramatic advances in CGM, the majority of people with diabetes in the world rely primarily on intermittent self-monitoring of blood glucose (SMBG). Furthermore, due to the cost of GM, even regular insulin users sometimes choose to omit GM some days. Efforts to reduce the user cost of GM, other than by governmental intervention, are unfortunately rare. ${ }^{43}$ Accurate SMBG devices are now widely available; greater usage of these while decreasing the usage of lessaccurate devices would be a welcome development. ${ }^{44}$

\section{CGM advances}

A primary focus of research and development in CGMs has been to improve accuracy and reduce or eliminate the number of user calibrations required to achieve this accuracy. ${ }^{45}$ Additional targets include ease of use, longer wear, miniturization, more impactful data management, and secure and reliable connectivity with the cloud and other devices.

Multiple additional improvements have been explored for CGMs, ranging from predictive glucose alerts to integration with AP systems. Other CGM improvements are being explored. Current CGM trend arrows are potentially useful, but neither the implementation of the arrows nor how they should be responded to has been standardized. Guidelines for the use of trend arrows have been published, ${ }^{46,47}$ but these focus on a single CGM system and are complex. Arrows are gradually being replaced with 30 to 60 min predicted glucose values; taking positive or negative glucose trend lines into account may help to standardize bolus doses once a CGM system is demonstrated to be capable of sufficiently accurate predictions. This would enable safer and more accurate adjustments to bolus doses and potentially reduce hypoglycemic and hyperglycemic events. ${ }^{4}$

Factory calibration for CGMs eliminates user calibration errors, improves convenience, reduces the expense of meterbased calibrations, and potentially improves sensor accuracy. ${ }^{49}$ One company currently developing an AP system reportedly chose a factory-calibrated CGM due to concerns about the potential inaccuracy introduced through user calibration errors. ${ }^{50}$ Although zero calibrations might appear to be ideal, factory-calibrated sensors might occasionally benefit from meter calibration if readings from an accurate meter significantly differed from those of the sensor. Redundancy with two to five working electrodes on each sensor can improve accuracy, with the additional advantage of allowing readings from a faulty sensor to be removed from the glucose average. ${ }^{51}$ Some current sensors may display inaccurate readings with the presence of acetaminophen, ascorbic acid (vitamin C), and acetylsalicylic acid. Dexcom G6 has eliminated acetaminophen interference, and other future sensors are expected to be more resistant to interfering substances.

Until recently, CGMs required a separate component ("receiver") to display glucose data. The FDA's increased regulatory flexibility allows smartphones to replace dedicated CGM receivers. Dexcom currently has the G5 mobile phone app (both iOS and Android) and Medtronic has the Guardian Connect app. Abbott has Libre Link and Libre LinkUp software (iOS and Android; currently not available in the United States). Using a smartphone adds both convenience and connectivity. Current Medicare regulations prevent seniors with diabetes from using connectivity apps to share data, even though this increases safety for seniors who live alone, have impaired cognition, or are experiencing glucose variability. This restriction is expected to be updated.

\section{Longer lasting implanted CGMs}

Senseonics recently received FDA approval for its 90-day implanted Eversense CGM (already approved in EU for 180 days). This small sensor can be inserted with a simple, $<5$-min office procedure. An external transmitter is worn on the skin and can be easily removed and reapplied, allowing for more user flexibility and convenience. Two daily calibrations yield a Mean Absolute Relative Difference (MARD) of $8.8 \% .^{52}$ Glysens has completed two trials with its 12-month sensor, implanted without general anesthesia in an outpatient surgery center. ${ }^{53,54}$ Both of these devices may offer additional options to those who decline to use CGM or have stopped using CGM due to tolerability issues. ${ }^{55}$

\section{Insulin Delivery Devices}

Although 2017 saw the exit of two companies (Animas and Roche) from the U.S. market, there are many innovations in pump therapy in the pipeline. These include an updated Insulet Omnipod pump (notable for greater connectivity, user interface improvements, compatibility with concentrated insulins, and AP functionality), Cellnovo's patch pump system currently obtaining FDA approval (notable for a unique pump mechanism using heat expansion of wax, cell network connectivity, and multiple AP collaborations), and a new pump from Eli Lilly for use in AP systems, also pending FDA approval. New entries targeted to type 2 diabetes are expected from BD and Calibra.

\section{Infusion sets}

With the availability of increasing number of "patch" pumps in the years ahead, standard long-line infusion sets may be replaced by automated insertion cannulas. Standard luer lock connections are also disappearing as companies transition to proprietary connections in their infusion sets. Of particular interest are efforts to increase the reliability and duration of wear of infusion sets. A revised BD Flowsmart ${ }^{\mathrm{TM}}$ infusion set is in development with secondary opening to reduce silent occlusions. A novel catheter that resists kinking and also has multiple holes is under investigation by Capillary Biomedical.

Efforts to combine a CGM sensor with an insulin infusion set have been complicated by the disparate wear times 
(typically longer for a sensor than an infusion set). Longer infusion set life and less-expensive sensors could make combined products more feasible. Initial concerns that proximity of insulin infusion near tissues in which glucose sensing was performed have been largely mollified. ${ }^{56}$ Questions concerning this remain, however, in regard to the impact of larger boluses or novel insulins.

\section{Smart insulin pens}

Today's smart Bluetooth insulin pens bring bolus calculators and data tracking to MDI patients. Companion Medical's Bluetooth LE reusable computerized InPen was approved by the FDA in July 2016. It features a bolus calculator, realtime insulin-on-board tracking, dose history data, reminders to avoid missed meal insulin doses, and an insulin temperature monitor. The InPen app also receives CGM data (Dexcom) and provides 24-h glucose averages and summary trend lines. Clinicians no longer remain in the dark regarding the insulin doses actually delivered. Users can set the app to automatically send a text message with each insulin dose, glucose reading, or carbohydrate entry to as many as five recipients.

In addition to its AP project, Bigfoot Biomedical is developing a Bluetooth insulin pen that connects to the FreeStyle Libre and a mobile app to monitor and automatically adjust long- and short-acting insulin doses. Pivotal trials for their smart insulin pen are expected to begin in 2018. Biocorp's Datapen is a smart reusable injector pen with audio alerts, dose tracking, and Bluetooth-enabling cloud connections, reminders, and advice. Biocorp and Common Sensing (Gocap) have add-on modules designed to fit over current "nonsmart" pens to extend these benefits to people using legacy pen injectors.

\section{Data Management, Decision Analysis, and Connected Care}

Computerized diabetes data management became widely available and was promoted by SMBG meter manufacturers in the late 1980s. Despite its potential utility, few clinicians or patients adopted it. Algorithms for diabetes care and insulin adjustment $^{57}$ were developed, but the obvious next step of implementing them in computerized systems was not realized commercially. The mechanics and time-consuming nature of uploading/downloading data from various devices had posed, and continue to pose, significant barriers. Diabetes telemedicine projects demonstrated benefit but were costly and could not attract sustained funding.

Since that time, the following key innovations were introduced: improved device connectivity (e.g., USB, Bluetooth), the Internet (and the "cloud"), and the connected smartphone and app. The connectivity and computing power embodied in these three technologies dramatically increased the reach (through the ubiquitous smartphone) and decreased the cost of diabetes data sharing. Automated uploads through the cloud minimize effort on the part of the patient and clinician, enabling near-universal, real-time access to data.

The ability of a diabetes-specific device to communicate with a smartphone enables the creation of a powerful platform for remote monitoring and advice. It also permits various independently developed proprietary modules to interact in a system that assists patients in both real time and longitudinally across episodes of care. We are just now on the brink of its practical and wide application in diabetes. However, due to the intense interest in this field, there is presently a bewildering plethora of early stage choices available to patients and clinicians.

Thousands of diabetes apps of unverified quality and safety are available for smartphones with little or no oversight. ${ }^{58}$ Glooko has emerged as the leading commercial integrator (with Tidepool the nonprofit competitor) of data from disparate diabetes devices. Rich silos of valuable proprietary cloudbased data from key device manufacturers (e.g., Medtronic, Dexcom, and Abbott) are beginning to share common formats (e.g., ambulatory glucose profile) and data. Catalyzing this progress is a new regulatory openness by the FDA with regard to software interoperability and the DIY (do it yourself) diabetes movement. The DIY AP project (https://openaps.org) is an example of open-source collaboration providing more advanced (although not fully tested), customizable, and modular functionality than is currently available commercially.

Artificial intelligence (AI) and machine learning are being applied to diabetes management. ${ }^{59}$ Medical devices and diagnostics tools are starting to provide blood glucose management tools that improve adherence. Medtronic's Sugar.IQ ${ }^{\mathrm{TM}}$ smart diabetes assistant is now available in the Guardian Connect CGM for people using MDIs. This AI technology from IBM Watson Health analyzes the relationship between glucose levels, food intake, insulin dosages, and other factors, and provides therapy suggestions. Early research showed a $2.6 \%$ increase in time-in-range and less hypoglycemia with $60 \%$ of participants using it daily. ${ }^{60}$

Machine learning uses pattern recognition and computational learning theory from AI to enable computers to construct algorithms that learn and make predictions from data, especially in situations like diabetes, wherein performance with standard software algorithms becomes difficult or infeasible. Based on data inputs that are becoming increasingly automated, a unique and individualized model is used to make predictions and decisions. Programmed hypo- and hyperalerts within a CGM system inform the wearer when glucose values drift above or below a desired glucose range and that corrective action may be warranted. Although "false alarms" can and do occur, the benefit of alerts to notify the wearer of possible need for intervention can be life saving.

What was known as "telemedicine", "telehealth", or "mhealth" is now being reimagined and renamed as "connected health"61 or "connected care." Communication among devices, clinicians, and patients including remote coaching is now increasingly possible; however, no one platform is mature or dominant.

The spectrum of options and opportunities in diabetesconnected care cannot be covered in this review. However, the following attributes can be used as a framework to classify, understand, and choose the appropriate technology: compatibility (which devices? which electronic medical record system?); interoperability and modularity (i.e., can I choose my insulin adjustment algorithm?); reliability, quality, data security, usage policies, ownership, business model, and cost.

The Blockchain is the newest potential fundamental ingredient of future connected care systems. More popularly known as the technology behind Bitcoin, the Blockchain can accomplish far more than just replacing cash with a digital currency. 
Each block in a medical Blockchain holds specific data owned by a patient that can be securely stored and transmitted among people, devices, and electronic medical record systems. Rather than having to access one's data from multiple healthcare providers who themselves cannot access data in a different EMR, the patient grants access to appropriate blocks of their data to a healthcare providers, insurers, or other approved parties. A Blockchain system allows healthcare-related data to be more easily accessible among healthcare providers, leading to better and faster treatment. It can contain messages and even software, while retaining its capacity to also act as a currency like Bitcoin. Although existing healthcare entities might view this as a threat, innovative organizations are developing new business models and variants of the Blockchain to enable new novel models of care.

\section{Conclusion}

New therapies, monitoring, and revolutionary-enabling technologies applied to healthcare represent an historic opportunity to improve the lives of people with diabetes. New medications and methods for their delivery are quickly becoming more effective. More meaningful monitoring of blood glucose values will occur with newer more useful devices more likely to be worn with more actionable information. Diabetes data need to be shared among patients, clinicians, devices, and other electronic systems.

Ubiquitous mobile devices with modular and interoperable software coupled with new technology, such as machine learning and Blockchains, will power connected health for diabetes. Connected care will replace more expensive, less convenient face-to-face clinic visits by enabling new models of care that increase velocity-to-control with more aggressive and frequent interventions that speed the achievement of glycemic goals. These new models of care must be effective, ethical, convenient, and financially sustainable.

\section{Author Disclosure Statement}

The authors declared the following potential conflicts of interest with respect to the research, authorship, and/or publication of this article: T.S.B. has received research support from Abbott, Ambra, Ascensia, BD, Boehringer Ingelheim, Calibra Medical, Companion Medical, Dance Biopharm, Dexcom, Eli Lilly, Glooko, Glysens, Kowa, Lexicon, MannKind, Medtronic, Novo Nordisk, Sanofi, Senseonics, Taidoc, Versartis, Xeris; consulting honoraria from Abbott, Astra Zeneca, Ascensia, BD, Calibra, Capillary Biomedical, Eli Lilly, Intarcia, Medtronic, Novo Nordisk, Sanofi; and speaking honoraria from Abbott, Eli Lilly, Medtronic, Novo Nordisk, and Sanofi. J.W. is a consultant for a range of companies that develop insulin pumps, bolus advisors, and infusion sets for the treatment of diabetes. He is employed by Advanced Metabolic Care and Research, which conducts numerous studies in diabetes devices, AP systems, medications, infusion sets, and insulins. J.Y.S. has received consulting honoraria from Senseonics.

\section{References}

1. World Health Organization: Global Report on Diabetes Geneva, 2016.
2. Hsu WC: Consequences of delaying progression to optimal therapy in patients with type 2 diabetes not achieving glycemic goals. South Med J 2009;102:67-76.

3. Fuller $\mathrm{H}$, Alberti $\mathrm{H}$ : Barriers to lifestyle changes in people with diabetes. Br J Gen Pract 2017;67:61.

4. Penn L, Dombrowski SU, Sniehotta FF, White M: Participants' perspectives on making and maintaining behavioural changes in a lifestyle intervention for type 2 diabetes prevention: a qualitative study using the theory domain framework. BMJ Open 2013;3:1-10.

5. Buysman EK, Anderson A, Bacchus S, Ingham M: Retrospective study on the impact of adherence in achieving glycemic goals in type 2 diabetes mellitus patients receiving canagliflozin. Adv Ther 2017;34:937-953.

6. Brown MT, Bussell JK: Medication adherence: WHO cares? Mayo Clin Proc 2011;86:304-314.

7. Cavalot F: Do data in the literature indicate that glycaemic variability is a clinical problem? Glycaemic variability and vascular complications of diabetes. Diabetes Obes Metab 2013;15 Suppl 2:3-8.

8. Suh S, Kim JH: Glycemic variability: how do we measure it and why is it important? Diabetes Metab J 2015;39: 273-282.

9. Monnier L, Colette C, Owens DR: Glycemic variability: the third component of the dysglycemia in diabetes. Is it important? How to measure it? J Diabetes Sci Technol 2008;2:1094-1100.

10. Beck RW, Connor CG, Mullen DM, et al.: The fallacy of average: how using $\mathrm{HbA} 1 \mathrm{c}$ alone to assess glycemic control can be misleading. Diabetes Care 2017;40:994-999.

11. Novo Nordisk: Novo Nordisk Receives FDA Approval for Fiasp $^{\circledR}$, a New Fast-Acting Mealtime Insulin. Plainsboro, NJ: PRNewswire, 2017.

12. Novo Nordisk: Novo Nordisk Launches Ozempic ${ }^{\circledR}$ and Fiasp $^{\circledR}$, Expanding Treatment Options for Adults with Diabetes. Ozempic ${ }^{\circledR}$, a new once-weekly GLP-1 RA and Fiasp ${ }^{\circledR}$, a New Fast-Acting Mealtime Insulin Are Now Available in U.S. Pharmacies. Plainsboro, NJ: PRNewswire, 2018.

13. Heise T, Pieber TR, Danne T, et al.: A pooled analysis of clinical pharmacology trials investigating the pharmacokinetic and pharmacodynamic characteristics of fast-acting insulin aspart in adults with type 1 diabetes. Clin Pharmacokinet 2017;56:551-559.

14. Biester T, Kordonouri O, Danne T: Pharmacological properties of faster-acting insulin aspart. Curr Diab Rep 2017;17: 101.

15. Russell-Jones D, Bode BW, De Block C, et al.: Fast-acting insulin aspart improves glycemic control in basal-bolus treatment for type 1 diabetes: results of a 26-week multicenter, active-controlled, treat-to-target, randomized, parallelgroup trial (onset 1). Diabetes Care 2017;40:943-950.

16. Bowering K, Case C, Harvey J, et al.: Faster aspart versus insulin aspart as part of a basal-bolus regimen in inadequately controlled type 2 diabetes: the onset 2 trial. Diabetes Care 2017;40:951-957.

17. Basu A, Pieber TR, Hansen AK, et al.: Greater early postprandial suppression of endogenous glucose production and higher initial glucose disappearance is achieved with fast-acting insulin aspart compared with insulin aspart. Diabetes Obes Metab 2018; doi: 10.1111/dom.13270.

18. Zijlstra E, Demissie M, Graungaard T, et al.: Investigation of pump compatibility of fast-acting insulin aspart in subjects with type 1 diabetes. J Diabetes Sci Technol 2018;12: 145-151. 
19. Heise T, Zijlstra E, Nosek L, et al.: Pharmacological properties of faster-acting insulin aspart vs insulin aspart in patients with type 1 diabetes receiving continuous subcutaneous insulin infusion: a randomized, double-blind, crossover trial. Diabetes Obes Metab 2017;19:208-215.

20. Bode BW, Johnson JA, Hyveled L, et al.: Improved postprandial glycemic control with faster-acting insulin aspart in patients with type 1 diabetes using continuous subcutaneous insulin infusion. Diabetes Technol Ther 2017;19: 25-33.

21. O'Meara A: Eli Lilly's New Ultra-Rapid Insulin in the Works. 2017. https://asweetlife.org/eli-lillys-new-ultra-rapidinsulin-in-the-works/ (accessed March 12, 2018).

22. Rege NK, Phillips NFB, Weiss MA: Development of glucoseresponsive 'smart' insulin systems. Curr Opin Endocrinol Diabetes Obes 2017;24:267-278.

23. Mohammadi D: Towards a smarter insulin. Pharm. J. 2017; 299.

24. Chou DH, Webber MJ, Tang BC, et al.: Glucose-responsive insulin activity by covalent modification with aliphatic phenylboronic acid conjugates. Proc Natl Acad Sci U S A 2015;112:2401-2406.

25. Monash University: Monash awarded \$1.1M grant to support development of revolutionary type of insulin. https://www .monash.edu/medicine/news/latest/2016-articles/46-milliongrant-to-support-development.html (accessed March 11, 2018).

26. Chen Z, Wang J, Sun W, et al.: Synthetic beta cells for fusion-mediated dynamic insulin secretion. Nat Chem Biol. 2018;14:86-93.

27. Heinemann L: The failure of exubera: are we beating a dead horse? J Diabetes Sci Technol. 2008;2:518-529.

28. Kraft KS, Grant M: Preparation of macromolecule-containing dry powders for pulmonary delivery. Methods Mol Biol 2009; 480:165-174.

29. Ray A, Mandal A, Mitra AK: Recent patents in pulmonary delivery of macromolecules. Recent Pat Drug Deliv Formul 2015;9:225-236.

30. Klonoff DC: Afrezza inhaled insulin: the fastest-acting FDA-approved insulin on the market has favorable properties. J Diabetes Sci Technol 2014;8:1071-1073.

31. Angelo R, Rousseau K, Grant M, et al.: Technosphere insulin: defining the role of Technosphere particles at the cellular level. J Diabetes Sci Technol 2009;3:545554.

32. Heinemann L, Baughman R, Boss A, Hompesch M: Pharmacokinetic and pharmacodynamic properties of a novel inhaled insulin. J Diabetes Sci Technol 2017;11:148156.

33. Afrezza: Prescribing Information. Danbury, CT: MannKind Corporation, 2014.

34. Mikhail N: Place of technosphere inhaled insulin in treatment of diabetes. World J Diabetes 2016;7:599-604.

35. Carls GS, Tuttle E, Tan RD, et al.: Understanding the gap between efficacy in randomized controlled trials and effectiveness in real-world use of GLP-1 RA and DPP-4 therapies in patients with type 2 diabetes. Diabetes Care 2017;40:1469-1478.

36. Gedawy A, Martinez J, Al-Salami H, Dass CR: Oral insulin delivery: existing barriers and current counter-strategies. J Pharm Pharmacol 2018;70:197-213.

37. Novo Nordisk: R\&D Pipeline. 2018. https://www.novonordisk .com/rnd/rd-pipeline.html (accessed March 25, 2018).
38. Buckley T, Connor A, Borregaard J, et al.: Site of absorption of an oral formulation of semaglutide. Paper presented at American Diabetes Association (ADA) 77th Scientific Sessions; June 11, 2017; San Diego, CA, 2017.

39. Davies M, Pieber TR, Hartoft-Nielsen ML, et al.: Effect of oral semaglutide compared with placebo and subcutaneous semaglutide on glycemic control in patients with type 2 diabetes: a randomized clinical trial. JAMA 2017;318: 1460-1470.

40. Oramed Pharmaceuticals Inc: Pipeline. 2018. www.oramed .com/pipeline/ (accessed March 10, 2018).

41. Intarcia Therapeutics Inc: Pipeline. 2018. www.intarcia.com/ pipeline-technology/pipeline.html (accessed March 10, 2018).

42. Rosenstock J, Buse JB, Azeem R, et al.: Efficacy and safety of ITCA 650, a novel drug-device GLP-1 receptor agonist, in type 2 diabetes uncontrolled with oral antidiabetes drugs: the FREEDOM-1 trial. Diabetes Care 2018;41:333-340.

43. Gainey Wilson K, Ovington P, Dean D: A low-cost inkjetprinted glucose test strip system for resource-poor settings. J Diabetes Sci Technol 2015;9:1275-1281.

44. Klonoff DC, Lias C, Beck S, et al.: Development of the diabetes technology society blood glucose monitor system surveillance protocol. J Diabetes Sci Technol 2016;10:697-707.

45. Bailey TS, Grunberger G, Bode BW, et al.: American Association of Clinical Endocrinologists and American College of Endocrinology 2016 outpatient glucose monitoring consensus statement. Endocr Pract 2016;22:231-261.

46. Aleppo G, Laffel LM, Ahmann AJ, et al.: A practical approach to using trend arrows on the Dexcom G5 CGM system for the management of adults with diabetes. J Endocr Soc 2017;1:1445-1460.

47. Laffel LM, Aleppo G, Buckingham BA, et al.: A practical approach to using trend arrows on the Dexcom G5 CGM system to manage children and adolescents with Diabetes. J Endocr Soc 2017;1:1461-1476.

48. Walsh J, Roberts R, Bailey TS, Heinemann L: Bolus advisors: sources of error, targets for improvement. J Diabetes Sci Technol 2018;12:190-198.

49. Hoss U, Budiman ES: Factory-calibrated continuous glucose sensors: the science behind the technology. Diabetes Technol Ther 2017;19:S44-S50.

50. Lagasse J: Abbott, Bigfoot Biomedical collaborate on diabetes technologies. Www.mobihealthnews.com/content/ abbott-bigfoot-biomedical-collaborate-diabetes-technologies (accessed March 17, 2018).

51. Castle JR, Ward WK: Amperometric glucose sensors: sources of error and potential benefit of redundancy. J Diabetes Sci Technol 2010;4:221-225.

52. Christiansen MP, Klaff LJ, Brazg R, et al.: A prospective multicenter evaluation of the accuracy of a novel implanted continuous glucose sensor: PRECISE II. Diabetes Technol Ther 2018;20:197-206.

53. Lucisano JY, Routh TL, Lin JT, Gough DA: Glucose monitoring in individuals with diabetes using a long-term implanted sensor/telemetry system and model. IEEE Trans Biomed Eng 2017;64:1982-1993.

54. ClinicalTrials.gov [Internet]. Bethesda (MD): National Library of Medicine (US). 2000-Identifier NCT02345967, Function of Implanted Glucose Sensor 2. https://Clinical Trials.gov/show/NCT02345967 (accessed March 31, 2018).

55. Engler R, Routh TL, Lucisano JY: Adoption barriers for continuous glucose monitoring and their potential reduction 
with a fully implanted system: results from patient preference surveys. Clin Diabetes 2018;36:50-58.

56. Ward WK, Castle JR, Jacobs PG, Cargill RS: Can glucose be monitored accurately at the site of subcutaneous insulin delivery? J Diabetes Sci Technol 2014;8:568574.

57. Skyler JS, Skyler DL, Seigler DE, O'Sullivan MJ: Algorithms for adjustment of insulin dosage by patients who monitor blood glucose. Diabetes care 1981;4:311318.

58. Huang Z, Soljak M, Boehm BO, Car J: Clinical relevance of smartphone apps for diabetes management: a global overview. Diabetes Metab Res Rev 2018:e2990.

59. Buch V, Varughese G, Maruthappu M: Artificial intelligence in diabetes care. Diabet Med 2018;35:495-497.
60. Agrawal P, Zhong A, Phukan A, et al.: Sugar.IQ insights: an innovative personalized machine-learning model for diabetes management. Diabetes 2017;66 (suppl 1):A63.

61. Kvedar J, Coye MJ, Everett W: Connected health: a review of technologies and strategies to improve patient care with telemedicine and telehealth. Health Aff (Millwood) 2014; 33:194-199.

Address correspondence to: Jenine Y. Stone, FNP-C AMCR Institute 625 West Citracado Parkway, Suite 112 Escondido, CA 92025

E-mail: jyagerstone@amcrinstitute.com 